\title{
Propiedades psicométricas de la escala de afrontamiento ante la ansiedad e incertidumbre pre-examen en universitarios chilenos
}

\section{Psychometric properties of the coping with pre-exam anxiety and uncertainty scale in chilean university students}

\section{Felipe Guerra Díaz*}

Universidad de Atacama, Copiapó, Chile

ORCID: https://orcid.org/0000-0003-0636-2566

Ricardo Jorquera Gutiérrez

Universidad de Atacama, Copiapó, Chile

ORCID: https://orcid.org/0000-0002-7059-8488

*Correspondencia:

Email: felipe.guerra@uda.cl
Citar como:

Guerra, F. \& Jorquera, R. (2021). Propiedades psicométricas de la escala de afrontamiento ante la ansiedad e incertidumbre pre-examen en universitarios chilenos. Propósitos y Representaciones, 9(3), e813. http://dx.doi.org/10.20511/pyr2021.v9n3.813 


\section{Resumen}

El presente estudio de tipo instrumental tuvo como objetivo describir las propiedades psicométricas de la Escala de Afrontamiento ante la Ansiedad e Incertidumbre Pre-Examen (COPEAU-A) en universitarios chilenos. Participaron 230 estudiantes de una universidad del norte de Chile, los cuales cursaban el primer año, donde un 67,4\% eran mujeres y un $32,6 \%$ hombres, con un promedio de edad de 18,9 años. Mediante análisis factorial confirmatorio, usando un método de mínimos cuadrados ponderados robusto (WLSMV), se compararon cinco modelos, encontrándose los mejores indicadores de ajuste en el compuesto por cuatro factores oblicuos con 20 ítems (X2=228,795, gl=164, p<,01; X2/gl= 1,395; TLI=,929; CFI=,939; RMSEA=,042). La confiabilidad de los cuatro factores fue adecuada, mostrando coeficientes Omega de McDonald entre $\omega=, 716$ y $\omega=, 873$. A su vez, los resultados evidenciaron la invarianza factorial del instrumento según sexo. Se concluye que las propiedades psicométricas del COPEAU-A apoyarían su uso para evaluar las estrategias de afrontamiento ante la ansiedad y la incertidumbre pre-examen en estudiantes chilenos que están iniciando sus carreras académicas en la universidad.

Palabras clave: Estrategias de afrontamiento; Ansiedad; Exámenes; Universitarios.

\section{Summary}

The present instrumental study aimed to describe the psychometric properties of the Coping with Pre-Exam Anxiety and Uncertainty Scale (COPEAU-A) in Chilean university students. 230 firstyear students from a university in the north of Chile participated, where $67,4 \%$ were women and $32.6 \%$ men, with an average age of 18,9 years. By confirmatory factor analysis, using a robust weighted least squares method (WLSMV), five models were compared, finding the best fit indicators in the composite of four oblique factors with 20 ítems $(X 2=228,795, g l=164, p<, 01$; $\mathrm{X} 2 / \mathrm{gl}=1,395 ; \mathrm{TLI}=, 929$; $\mathrm{CFI}=, 939$; RMSEA=,042). The reliability of the four factors was adequate, showing McDonald's Omega coefficients between $\omega=, 716$ and $\omega=, 873$. In turn, the results showed the factorial invariance of the instrument according to sex. It is concluded that the psychometric properties of COPEAU-A would support its use to evaluate coping strategies in the face of anxiety and pre-exam uncertainty in Chilean university students who are beginning their academic careers.

Keywords: Coping strategies; Anxiety; Exams; University.

\section{Introducción}

El ingreso a la universidad genera un proceso de cambio en los estudiantes, donde las diversas actividades académicas que se presentan, las modificaciones en la rutina, la inquietud por el rendimiento y el malestar físico que aparece en este contexto universitario, podrían asociarse a ansiedad ante las evaluaciones (AE) (Angelucci, Cañoto \& Hernández, 2017). Se entiende la AE como la expresión de síntomas conductuales, fisiológicos y cognitivos (Oermann \& Gaberson, 2009), que pueden presentarse en el momento mismo de la rendición de una evaluación académica, así como antes o posterior a la misma, viviendo las evaluaciones académicas como una circunstancia inquietante y dañina, centrada en la aprensión hacia la no obtención de los réditos esperados (Fernández-Castillo \& Caurcel, 2015).

La rendición de evaluaciones académicas tiene gran importancia en el desarrollo de las carreras de los estudiantes, por lo tanto, se hace primordial contar con respuestas adecuadas para hacer frente a las demandas de la vida universitaria, las cuales deberían estar en sintonía con el logro de los objetivos propuestos (Zeidner, 1995). Estas respuestas, comportamentales y cognitivas, que se llevan a cabo para regular las diversas exigencias internas y/o del medio que 
son evaluadas como superiores a las capacidades y recursos con los que cuenta el sujeto, son las que se conocen como estrategias de afrontamiento (Lazarus \& Folkman, 1984).

Considerando que pueden existir diversas respuestas de los sujetos a las demandas del medio, las estrategias de afrontamiento pueden ser clasificadas en tres (Zeidner, 1995): a) estrategias orientadas al problema, se basan en dar solución a la dificultad que está emergiendo para así poder contrarrestar el estrés, donde destacan la planificación estratégica, aumento del tiempo de estudio, la reinterpretación de la situación, eliminar acciones distractoras, aceptación de la situación y el afrontamiento activo; b) afrontamiento orientado a la emoción, las cuales buscan regular el estrés emocional asociado al problema, donde se encuentran la búsqueda de apoyo social por razones emocionales y por razones instrumentales, la expresión emocional, el diálogo con otros, técnicas de relajación y respiración; y c) afrontamiento orientado a la evitación, basadas en sortear el acontecimiento que genera el estrés a través de la negación, desapego conductual y mental, humor y consumo de sustancias principalmente.

El afrontamiento en las evaluaciones académicas, así como variadas situaciones estresantes del día a día de los sujetos se componen de cuatro fases: a) fase anticipatoria, se da antes de la evaluación académica, donde el estudiante se prepara para rendir la evaluación y se preocupa en relación a qué hacer para afrontar dicha situación; b) fase de confrontación, en esta fase los estudiantes rinden la evaluación académica y es el momento donde la ansiedad aumenta y donde los estudiantes utilizan diversas estrategias de afrontamiento, ya sea centradas en la emoción y/o centradas en el problema; c) fase de espera, en esta la evaluación académica ya ha sido rendida por parte de los estudiantes, pero aún no conocen el resultado; y d) fase de resultados, posterior a que los estudiantes conocen las calificaciones obtenidas, se realiza el análisis respecto al rendimiento obtenido, la incertidumbre desaparece, pero pueden aparecer inquietudes en cuanto a lo relevante de lo acontecido y si esto generó ganancias o pérdidas (Lazarus \& Folkman, 1984, Carver \& Scheier, 1995).

En relación a la medición de las estrategias de afrontamiento en evaluaciones académicas, el Coping with Pre-Exam Anxiety and Uncertainty (COPEAU) de Stöber (2004) es un instrumento creado en específico para determinar las estrategias de afrontamiento utilizadas antes de la rendición de las evaluaciones académicas, que toma como base dos cuestionarios previos, el COPE (Carver, Scheier, \& Weintraub, 1989) y las subescalas de afrontamiento del Inventario Diferencial de Ansiedad frente a los Exámenes (DAI) (Rost \& Schermer, 1997). Este instrumento está compuesto por 3 dimensiones del afrontamiento: a) Orientación a la tarea y preparación, b) búsqueda de apoyo social, y c) evitación, cada una con 7 ítems. Fue elaborado a partir del análisis de componentes principales que arrojó cuatro factores con autovalores superiores a 1. Posteriormente, se realizó un análisis paralelo con rotación de tipo ortogonal, llegando a un modelo de tres factores.

La adaptación al español del test COPEAU, realizada en población argentina con estudiantes universitarios, se conoce como COPEAU-A (Heredia, Piemontesi, Furlan \& Pérez, 2008), la que fue conseguida a través de la realización de un análisis con el método de componentes principales, arrojando una solución de 4 factores, lo cual fue coherente con la estructura presentada por el test COPE, al existir diferencias en la solución factorial los investigadores se contactan con Stöber y toman la propuesta planteada por el autor de conservar la estructura de 3 factores.

Con el objetivo de continuar evaluando las propiedades psicométricas de COPEAU-A, ahora en población universitaria peruana, donde participaron estudiantes de la carrera de psicología de primero a sexto año, Domínguez-Lara y Merino-Soto (2016) realizaron un análisis factorial confirmatorio que corroboró una estructura de 4 factores (orientación a la tarea, búsqueda de apoyo instrumental, búsqueda de apoyo emocional y evitación), con adecuados indicadores de consistencia interna. Los mismos autores propusieron una versión breve, llamada COPEAU-B, 
que evidenció una estructura factorial de 4 factores equivalente a la versión extensa, contando además con adecuados coeficientes de fiabilidad, lo cual avalaba las adecuadas propiedades psicométricas de la versión breve (Domínguez-Lara \& Merino-Soto, 2018).

Variados han sido hallazgos referidos a las estrategias de afrontamiento de la ansiedad pre-examen que se han encontrado usando el COPEAU-A. Domínguez-Lara (2018a) halló correlaciones estadísticamente significativas entre la autoeficacia académica percibida y las estrategias orientación a la tarea y evitación, con lo cual arribó a la conclusión que las personas que presentan un mayor nivel de autoeficacia académica se inclinarían por utilizar estrategias más relacionadas con el control de la situación anterior al examen, que las destinadas a buscar apoyo o a evitar el suceso. En otro estudio, Domínguez-Lara (2018b) buscó conocer la influencia de las estrategias de afrontamiento pre-examen (EAPE) sobre la ansiedad ante los exámenes-estado (AE Estado) en estudiantes de psicología, encontrando que las EAPE explican el 13,3\% de la varianza de la AE-Estado, siendo las estrategias de orientación a la tarea y la búsqueda de apoyo social por razones instrumentales las de mayor influencia. A su vez, Domínguez-Lara (2018c) encontró correlaciones estadísticamente significativas entre las dimensiones del COPEAU-A, procrastinación y rendimiento académico. Específicamente evidenció relación directa entre orientación a la tarea y la autorregulación académica, e inversa con la postergación de actividades, y además, encontró relación inversa entre evitación y autorregulación académica y directa con la postergación de actividades. A su vez, detectó que rendimiento académico general se relaciona directamente con estrategia orientada a la tarea e inversa con las demás dimensiones del COPEAU.

Santabárbara (2019) ocupó el COPEAU-A para describir las estrategias de afrontamiento a la ansiedad e incertidumbre utilizadas por los estudiantes de Medicina ante la rendición de un examen de bioestadística y de esta forma estimar su relación con el desempeño en la asignatura. El autor encontró que los estudiantes de medicina presentan estrategias de afrontamiento orientadas a la búsqueda de apoyo social y a la tarea, las cuales influyen de manera positiva en el rendimiento de dicha asignatura. Por su parte Arana y Furlan (2015), usando el COPEAU-A encontraron que los perfeccionistas que buscan altos estándares eran propensos a seleccionar estrategias de afrontamiento orientadas a la tarea.

A partir de las adecuadas propiedades psicométricas presentadas por el COPEAU-A en población universitaria argentina y peruana, y el no contar en Chile con un instrumento validado para estos fines, hace que se llegue al interés por indagar en sus características psicométricas con estudiantes universitarios de este país.

\section{Método}

\section{Diseño}

El objetivo de la presente investigación es la estimación de las propiedades psicométricas de un instrumento de evaluación, por ello este estudio es de tipo instrumental (Ato, López y Benavente, 2013). A su vez, el diseño de investigación es no experimental transversal-descriptivo.

\section{Participantes}

Se utilizó un muestreo no probabilístico intencional. La muestra estuvo constituida por 230 estudiantes de primer año pertenecientes a cinco facultades de una universidad pública del norte de Chile. De ellos, un 67,4\% fueron mujeres $(n=155)$ y un $32,6 \%$ hombres $(n=75)$, con edades comprendidas entre los 17 y 41 años (M=18,90; DE: 3,1). Dentro de los criterios de inclusión se consideró ser hombre y/o mujer matriculado/a en primer año de una carrera de la universidad. Por ello, se excluyeron a estudiantes no matriculados en la universidad en que se realizó el estudio o ser de un nivel superior. Todos participaron de forma absolutamente voluntaria. 


\section{Instrumento}

Se utilizó la adaptación al español de la escala de afrontamiento ante la ansiedad e incertidumbre pre-examen COPEAU (Stöber, 2004), validada en Argentina y denominada COPEAU-A (Heredia, Piemontesi, Furlan \& Pérez, 2008). Este instrumento es un auto reporte de 21 ítems distribuidos en tres dimensiones que evalúan tres estrategias de afrontamiento: Orientación a la Tarea y Preparación $(\alpha=.87)$, Búsqueda de apoyo social $(\alpha=.85)$, y Evitación $(\alpha=.70)$. El sistema de respuesta utiliza una escala tipo Likert de 6 puntos que van de 1- "Nunca lo hago" a 6- "Siempre lo Hago".

\section{Procedimientos}

Los datos fueron recolectados al inicio de una clase taller que tendía a la inserción de los estudiantes en la vida universitaria en una universidad pública del norte de Chile, con estudiantes de primer año, a los cuales se les pidió la colaboración anónima y voluntaria. A todos los participantes se les explicaron los fundamentos de la investigación, el resguardo y uso que se daría a la información obtenida. La totalidad de participantes firma un consentimiento informado. El test fue administrado de manera online y masiva a través de un formulario de Google, en momentos determinados para cada una de las carreras participantes.

\section{Análisis de datos}

En primer lugar, se estimaron los estadísticos descriptivos de los ítems, específicamente medias, desviaciones standard, asimetría y curtosis. Luego, se realizó un análisis factorial confirmatorio (AFC) para verificar la estructura del instrumento, lo cual se realizó mediante el método de mínimos cuadrados ponderados robusto (WLSMV), dado que no asume la distribución normal de las variables y es considerado adecuado para datos ordinales (Brown, 2015). En este procedimiento se estimaron los siguientes índices: $\mathrm{chi}^{2}, \mathrm{chi}^{2} / \mathrm{gl}$, el Índice de Tucker-Lewis (TLI) y el Índice de Bondad de Ajuste Comparativo (CFI). Se estiman como buenos indicadores de ajustes un $\mathrm{chi}^{2}$ no significativo, un valor $\mathrm{chi}^{2} / \mathrm{gl}$ inferior a 3 y valores superiores a ,90 en CFI y TLI. También se evaluó la raíz del residuo cuadrático promedio de aproximación (RMSEA), considerando como aceptables valores inferiores a ,08 (Hu \& Bentler, 1999). La confiabilidad del instrumento se constató con estadígrafo Omega de McDonald. Junto a lo anterior, se estimó la invarianza factorial del instrumento según género (mujeres y hombres). Para tal efecto, los indicadores de ajustes utilizados fueron los mismos usados en el AFC anterior, describiéndose y comparando de forma secuencial la invarianza configural, métrica, escalar y estricta del instrumento, observando que las variaciones en los valores de CFI entre cada procedimiento fueran inferiores a ,010 y que las variaciones de RMSEA fueran menores a ,015 (Byrne, 2008; Cheung \& Rensvold, 2002). Posteriormente, se procedió a comparar las medias de hombres y mujeres en los diversos factores del instrumento, lo cual se realizó mediante una prueba t de Student para muestras independientes. Para finalizar, mediante percentiles se establecieron los baremos interpretativos del instrumento para hombres y mujeres. Se usó el software SPSS 22 para efectuar los análisis descriptivos, las prueba t student y el cálculo de percentiles. Los análisis factorial confirmatorios y los análisis de invarianza factorial se realizaron con el software $\mathrm{R}$ versión 4.0.2 ( $\mathrm{R}$ Core Team, 2020) ejecutado en la terminal RStudio versión 1.3.1093. La estimación de la confiabilidad mediante el estadígrafo omega de McDonald's $(\omega)$ se realizó con el software JASP 0.14 (JASP Team, 2020).

\section{Resultados}

Primeramente, se efectuó el análisis descriptivo de los ítems. Al respecto, es posible señalar que las medias más altas se encontraron en los ítems "Concentro todos mis esfuerzos en el examen" $(\mathrm{M}=4,80$; $\mathrm{DS}=1,037)$, "Hago lo que se necesite para tener lista cada cosa en su momento" $(\mathrm{M}=4,47$; $\mathrm{DS}=1,096)$ y "Pienso cuál es la mejor forma de prepararme para el examen" $(\mathrm{M}=4,44$; DS $=1,187$ ), todos ellos pertenecientes al factor Orientación a la Tarea. Por su parte, las medias 
más bajas se observan en los ítems "Alejo los pensamientos acerca del examen de mi mente" $(\mathrm{M}=2,77 ; \mathrm{DS}=, 992)$ y "Trato de no pensar en el examen" $(\mathrm{M}=2,80 ; \mathrm{DS}=1,024)$, ambos del factor Evitación.

Evaluada la asimetría y curtosis, todos los reactivos del instrumento presentaron valores absolutos inferiores a 1 (ver Tabla 1).

Tabla 1.

Estadísticos descriptivos ítems

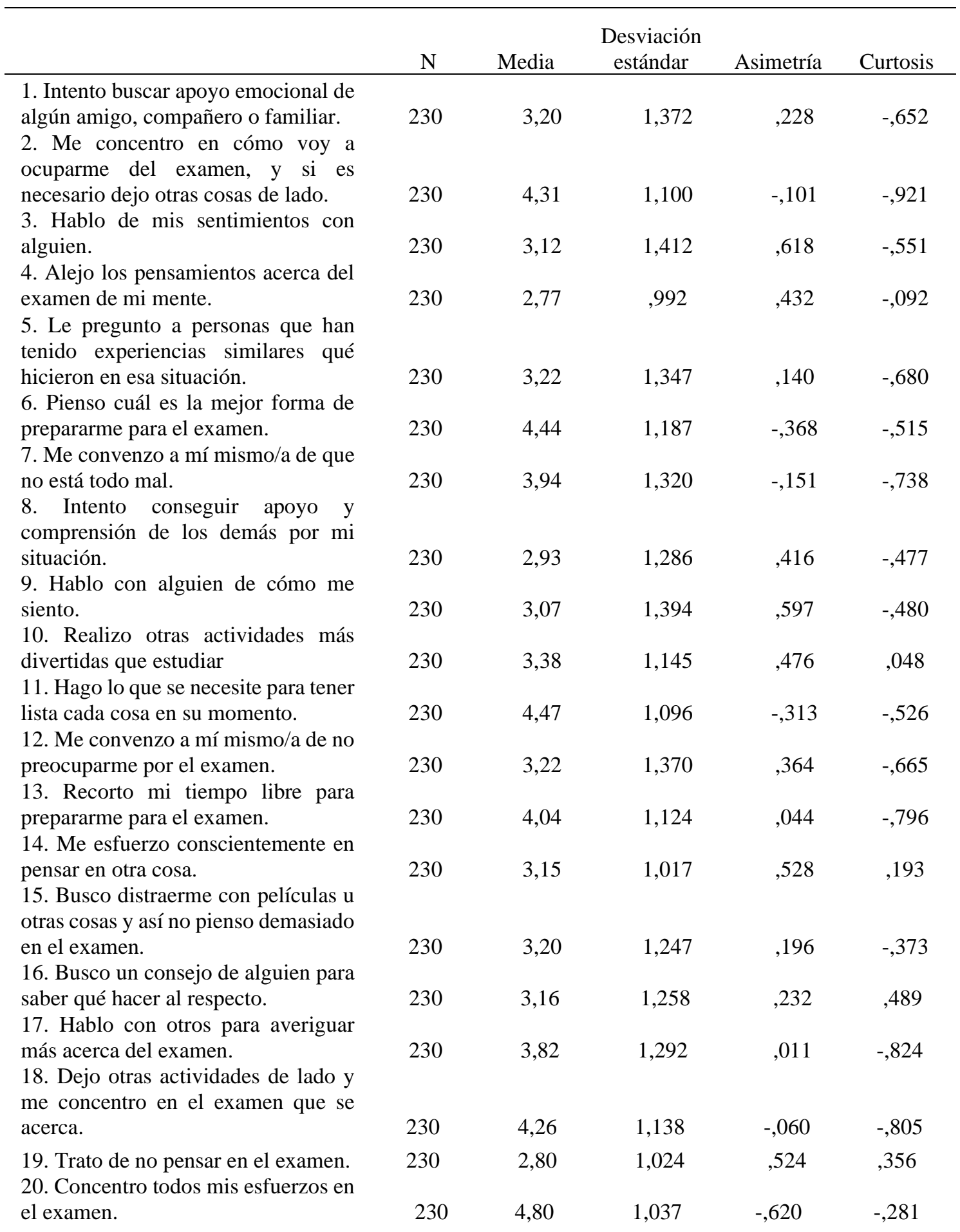


21. Me tomo tiempo extra para

prepararme para el examen.

230

4,07

1,152

$-, 059$

$-, 591$

La normalidad multivariada fue evaluada mediante un análisis Mardia para la asimetría y curtosis multivariada. Se evidenció un coeficiente de asimetría de 58,815 ( $\mathrm{X}^{2}=2254,581$, gl = $1540, \mathrm{p}<, 001)$ y un coeficiente de curtosis de 481,650 (z=10,647; p<,001), con lo que se constató la ausencia de una distribución normal multivariada de los datos. Dado lo anterior, y considerando también el sistema de respuesta tipo Likert del instrumento, se decidió desarrollar el análisis factorial confirmatorio a través del método de mínimos cuadrados ponderados robusto (WLSMV) (Brown, 2015).

Por medio de análisis factorial confirmatorio se verificaron cinco modelos. Las razones por las cuales se hace esto está relacionado con ciertas bases en que se sustentó el creador del instrumento, las cuales no lograron ser estimadas a nivel latinoamericano (especialmente Perú y Argentina). En ese sentido, en sus estimaciones originales Stöber (2004), asumió un modelo de 3 factores ortogonales (no relacionados). La adaptación hispanoparlante de Heredia et al. (2008) muestra una estructura de cuatro factores, que evidencian relación entre ellas. Esto último lo puntualizaría Domínguez-Lara y Merino Soto (2016) quienes señalan que teóricamente se puede argumentar que las estrategias de afrontamiento cavarían en algún grado entre ellas. Por esta razón se decidió probar la estructura original con las consideraciones de su autor y los nuevos hallazgos, tanto en lo que a estructura como a supuestos se refiere.

Por un lado, se probó el modelo original de Stöber (2004) conformado por tres factores: orientación hacia la tarea, evitación y búsqueda de apoyo. Este modelo se evaluó considerando la independencia de sus factores (ortogonales), y también asumiendo la relación entre ellos (oblicuos). Posteriormente, se verificó el ajuste del modelo de cuatro factores de Heredia et al. (2008), en donde se mantienen los factores originales orientación a la tarea y evitación de Stöber, pero dividiendo el factor búsqueda de apoyo en dos: búsqueda de apoyo por razones instrumentales y búsqueda de apoyo por razones emocionales. Este modelo también se evaluó considerando la independencia de los factores y asumiendo su relación. Finalmente se analizó el ajuste de este último modelo sin la pregunta siete, como lo propuso Domínguez-Lara y MerinoSoto (2016), quienes asumieron que los factores estaban asociados (oblicuos).

Los indicadores que fueron usados fueron $\mathrm{x}^{2} / \mathrm{gl}$, CFI, TLI y RMSEA. Considerando que $\mathrm{x}^{2}$ suele ser muy sensible al tamaño de la muestra se utilizó el valor de $\mathrm{x} 2 / \mathrm{gl}$. De acuerdo a este indicador, la literatura señala que un modelo demuestra un ajuste razonable si el estadístico ajustado por sus grados de libertad no excede 3 (Kline, 2005; Iacobucci, 2010). Por su parte, Hu y Bentler (1999) recomiendan valores superiores a ,90 en CFI y TLI para considerar un ajuste aceptable. A su vez, valores inferiores a ,08 en RMSEA se estima como aceptable (ver Tabla 2).

Tabla 2.

Indicadores de Ajuste de Modelos Medidos del COPEAU-A

\begin{tabular}{|c|c|c|c|c|c|c|c|}
\hline Modelo & $\begin{array}{c}X^{2} \\
\text { Robusto }\end{array}$ & df & $\mathrm{p}$ & $\mathrm{X}^{2} / \mathrm{df}$ & CFI & TLI & RMSEA \\
\hline 3 Factores ortogonales & 458,75 & 189 & ,000 & 2,427 & ,761 & 735 & $\begin{array}{l}, 079 \\
{[\text { IC90\% }=, 070-, 088]}\end{array}$ \\
\hline 3 Factores oblicuos & 362,605 & 186 &, 000 & 1,949 & ,844 &, 824 & $\begin{array}{l}, 064 \\
{[\mathrm{IC} 90 \%=, 054-, 074]}\end{array}$ \\
\hline 4 Factores ortogonales & 583,758 & 189 &, 000 & 3,089 & ,651 & ,612 & $\begin{array}{l}, 096 \\
{[\mathrm{IC} 90 \%=, 087-, 104]}\end{array}$ \\
\hline 4 Factores oblicuos & 346,587 & 183 &, 000 & 1,894 & ,855 &, 834 & $\begin{array}{l}, 062 \\
{[\text { IC } 90 \%=, 052-, 072]}\end{array}$ \\
\hline $\begin{array}{l}4 \text { Factores oblicuos } \\
\text { sin item } 7\end{array}$ & 228,795 & 164 & ,001 & 1,395 & ,939 & ,929 & $\begin{array}{l}, 042 \\
{[\mathrm{IC} 90 \%=, 028-, 054]}\end{array}$ \\
\hline
\end{tabular}

Propósitos y Representaciones

Set - Dic 2021, Vol. 9, $\mathrm{N}^{\circ} 3$, e813

http://dx.doi.org/10.20511/pyr2021.v9n3.813 
El modelo de tres factores evidenció indicadores de ajustes inferiores a los aceptables considerando el modelo ortogonal $\left(\mathrm{X}^{2}=458,75, \mathrm{gl}=189, \mathrm{p}<, 001 ; \mathrm{X}^{2} / \mathrm{gl}=2,427 ; \mathrm{TLI}=, 735\right.$; $\mathrm{CFI}=, 761 ; \mathrm{RMSEA}=, 079)$ y también el oblicuo $\left(\mathrm{X}^{2}=362,605, \mathrm{gl}=186, \mathrm{p}<, 001 ; \mathrm{X}^{2} / \mathrm{gl}=1,949\right.$; $\mathrm{TLI}=, 824 ; \mathrm{CFI}=, 844 ; \mathrm{RMSEA}=, 064)$. El modelo de cuatro factores propuesto por Heredia et al. (2008) mostró resultados bajos cuando se consideró de forma ortogonal $\left(\mathrm{X}^{2}=583,758, \mathrm{gl}=189\right.$, $\left.\mathrm{p}<, 001 ; \mathrm{X}^{2} / \mathrm{gl}=3,089 ; \mathrm{TLI}=, 612 ; \mathrm{CFI}=, 651 ; \mathrm{RMSEA}=, 096\right)$. Cuando se probó este modelo de forma oblicua mejoró sus indicadores, sin embargo, aun de forma insuficiente a lo adecuado $\left(\mathrm{X}^{2}=346,587, \mathrm{gl}=183, \mathrm{p}<, 001 ; \mathrm{X}^{2} / \mathrm{gl}=1,894 ; \mathrm{TLI}=, 834 ; \mathrm{CFI}=, 855 ; \mathrm{RMSEA}=, 062\right)$.

El modelo propuesto por Domínguez-Lara y Merino-Soto (2016), el cual difería del propuesto por Heredia et al. (2008) en el hecho de que descartaron una pregunta, evidenció mayor número de indicadores de ajustes en niveles aceptables. En este caso, el índice $\mathrm{X}^{2}$ resultó ser significativo $\left(X^{2}=228,795, \mathrm{gl}=164, \mathrm{p}<, 001\right)$, sin embargo, la razón $X^{2} / g l$ fue de 1,395 fue adecuada. Asimismo, el Índice de Tucker-Lewis (TLI=,929) y el Índice de Bondad de Ajuste Comparativo (CFI=,939) tuvieron valores aceptables, al igual que el Root Mean Square Error of Aproximation (RMSEA=,042).

Se calculó la confiabilidad de los cuatro factores del modelo de 20 ítems distribuidos en cuatro factores, evidenciándose indicadores omegas de McDonald's superiores a ,7 en todos ellos: orientación a la tarea $(\omega=, 868)$, evitación $(\omega=, 716)$, búsqueda de apoyo instrumental $(\omega=, 717)$ y búsqueda de apoyo emocional $(\omega=, 873)$ (ver Tabla 3$)$.

Tabla 3.

Pesos de Regresión Estandarizados, Confiabilidad y Análisis de Ítems modelo de 4 factores (20 items)

\begin{tabular}{|c|c|c|c|c|}
\hline Factor/Confiabilidad & Item & $\begin{array}{c}\text { Pesos factoriales } \\
\text { estandarizados }\end{array}$ & $\begin{array}{c}\omega \text { si el item } \\
\text { se elimina }\end{array}$ & $\begin{array}{l}\text { Correlación } \\
\text { Item/Factor }\end{array}$ \\
\hline \multirow{7}{*}{$\begin{array}{l}\text { Orientación a la Tarea } \\
\omega=, 868 \\
{[\text { CI95\%=,839- }-892]}\end{array}$} & Item 2 & ,646 &, 854 & ,598 \\
\hline & Item 6 & ,705 & ,856 & 600 \\
\hline & Item 11 & 676 & 853 & 616 \\
\hline & Item 13 & ,715 & ,843 & ,682 \\
\hline & Item 18 & ,783 & ,836 & ,729 \\
\hline & Item 20 & ,676 &, 850 & ,633 \\
\hline & Item 21 & ,663 & ,851 & ,626 \\
\hline \multirow{6}{*}{$\begin{array}{l}\text { Evitación } \\
\omega=, 716 \\
{[\text { CI95\%=,660 - ,773] }}\end{array}$} & Item 4 & ,384 & ,704 & ,322 \\
\hline & Item 10 & ,379 & ,715 & ,306 \\
\hline & Item 12 & 655 & 653 &, 542 \\
\hline & Item 14 &, 543 & 681 & ,439 \\
\hline & Item 15 & ,561 & 677 & ,494 \\
\hline & Item 19 & ,712 & 653 &, 531 \\
\hline \multirow{3}{*}{$\begin{array}{l}\text { Búsqueda de apoyo Instrumental } \\
\omega=, 717 \\
{[\mathrm{CI} 95 \%=, 634-, 767]}\end{array}$} & Item 5 & ,638 & ,556 &, 570 \\
\hline & Item 16 & ,882 &, 563 & ,568 \\
\hline & Item 17 & ,495 & ,716 & ,441 \\
\hline \multirow{4}{*}{$\begin{array}{l}\text { Búsqueda de apoyo Emocional } \\
\omega=, 873 \\
{[\text { CI95\%=,834 - ,892] }}\end{array}$} & Item 1 & ,714 & 879 & ,640 \\
\hline & Item 3 & ,809 & ,801 & ,786 \\
\hline & Item 8 & ,782 & ,878 & 622 \\
\hline & Item 9 & ,832 & ,786 &, 821 \\
\hline
\end{tabular}

La Tabla 4 muestra las correlaciones entre los factores del COPEAU-A. Se aprecia una correlación cercana a 0 entre orientación a la tarea y evitación. Por su parte, las relaciones entre orientación a la tarea y búsqueda de apoyo instrumental y emocional, y entre evitación y búsqueda 
de apoyo emocional, son de modestas (entre 255 y ,325). En contraste a lo anterior, búsqueda de apoyo emocional e instrumental muestran una correlación alta $(, 702)$.

Tabla 4.

Correlaciones entre los factores del COPEAU-A (20 items)

\begin{tabular}{lccc}
\hline & Evitación & $\begin{array}{c}\text { Búsqueda de apoyo } \\
\text { instrumental }\end{array}$ & $\begin{array}{c}\text { Búsqueda de apoyo } \\
\text { emocional }\end{array}$ \\
\hline Orientación a la tarea &, 057 &, 307 &, 255 \\
Evitación & &, 325 &, 262 \\
Búsqueda de apoyo instrumental & & &, 702 \\
\hline
\end{tabular}

\section{Invarianza Factorial por Sexo}

La invarianza factorial es un procedimiento en donde se verifica que las propiedades de medida de los instrumentos o sus ítems son independientes de las características de los grupos evaluadas, pero no del constructo que el instrumento está midiendo. Para ello evalúa sucesivamente la invarianza configural (referida a la estructura del instrumento entre los grupos), invarianza métrica (referida a las cargas factoriales entre los grupos), invarianza fuerte (referida a los interceptos entre los grupos) e invarianza estricta (referida a los residuos) mediante un Análisis Factorial Confirmatorio multigrupo (Byrne, 2008). Para la evaluación de las diferencias entre los modelos, se emplearon las variaciones de los índices CFI $(\Delta \mathrm{CFI})$ y RMSEA ( $\triangle$ RMSEA). Las invariancias se admitieron con valores $\Delta \mathrm{CFI} \leq 0.01$ y $\triangle$ RMSEA $\leq 0.015$ (Cheung \& Rensvold, 2002).

Se evaluó la invarianza factorial sexo del instrumento, considerando un modelo de cuatro factores y 20 ítems, dado que fue el que mostró mejores indicadores de ajuste.

En primer lugar, se analizó la invarianza configural del COPEAU (20 ítems), mostrando valores adecuados (CFI=,922; TLI=,910; RMSEA=,044). Se manifiesta que el modelo de cuatro factores se ajustaría a los datos en ambos grupos. Posteriormente, se evaluó la invarianza métrica apreciándose valores favorables ( $\mathrm{CFI}=, 930 ; \mathrm{TLI}=, 923 ; \mathrm{RMSEA}=, 040)$. Al comparar los resultados de la invarianza métrica y configural no se aprecian diferencias significativas $(\triangle \mathrm{CFI}=$ ,008 y $\triangle$ RMSEA = -,004). Los resultados permiten establecer que las cargas factoriales son invariantes entre ambos grupos. En tercer lugar, se evaluó la invarianza escalar. Los resultados muestran adecuados índices de ajuste (CFI=,931; TLI=,927; RMSEA=,039). Al comparar los resultados de la invarianza métrica y escalar no se constatan cambios importantes $(\Delta \mathrm{CFI}=, 001 \mathrm{y}$ $\triangle$ RMSEA $=-, 001$ ), lo que permite señalar que los interceptos son invariantes en los grupos de hombres y mujeres. Finalmente, se analizó la invarianza estricta constatándose adecuados índices de ajuste $(\mathrm{CFI}=, 933$; TLI=,933; RMSEA=,038). El cotejo de los índices de ajuste de la invarianza escalar muestra diferencias adecuadas $(\triangle C F I=, 002$ y $\triangle$ RMSEA $=-, 001)$, permitiendo entregar un soporte empírico a la invarianza estricta.

Los resultados permiten confirmar la invarianza factorial según sexo del COPEAU en su versión de 20 ítems (ver Tabla 5).

Tabla 5.

Índices de bondad de ajuste de los modelos puestos a prueba en la invarianza factorial

\begin{tabular}{|c|c|c|c|c|c|c|c|c|}
\hline & $\begin{array}{c}\mathrm{X}^{2} \\
\text { Robusto } \\
\end{array}$ & DF & $\mathrm{X}^{2} / \mathrm{DF}$ & CFI & TLI & RMSEA & $\Delta \mathrm{CFI}$ & $\triangle \mathrm{RMSEA}$ \\
\hline $\begin{array}{l}\text { Invarianza } \\
\text { Configural }\end{array}$ & 399,414 & 328 & 1,218 & ,922 & ,910 & $\begin{array}{c}, 044 \\
{[\mathrm{IC} 90 \%=, 026-, 058]}\end{array}$ & & \\
\hline $\begin{array}{l}\text { Invarianza } \\
\text { Métrica }\end{array}$ & 407,797 & 344 & 1,185 & ,930 & 923 & $\begin{array}{c}, 040 \\
{[\mathrm{IC} 90 \%=, 021-, 055]}\end{array}$ & ,008 &,- 004 \\
\hline
\end{tabular}




\begin{tabular}{|c|c|c|c|c|c|c|c|c|}
\hline $\begin{array}{l}\text { Invarianza } \\
\text { Escalar }\end{array}$ & 423,197 & 360 & 1,176 & ,931 & ,927 & $\begin{array}{c}, 039 \\
{[\mathrm{IC} 90 \%=, 020-, 054]}\end{array}$ & ,001 &,- 001 \\
\hline $\begin{array}{l}\text { Invarianza } \\
\text { Estricta }\end{array}$ & 441,554 & 380 & 1,162 & ,933 & ,933 & $\begin{array}{c}, 038 \\
{[\mathrm{IC} 90 \%=, 018-, 052]}\end{array}$ & ,002 &,- 001 \\
\hline
\end{tabular}

\section{Baremos}

La Tabla 6 describe los estadísticos de los puntajes de las dimensiones del instrumento, considerando el modelo de 4 factores y 20 preguntas. En este caso, únicamente se observa diferencias significativas entre hombres y mujeres en el factor búsqueda de apoyo emocional $(\mathrm{t}=2,948, \mathrm{p}<, 01)$, donde las mujeres evidencian medias más altas.

Tabla 6.

Descriptivos y comparación de medias de dimensiones de la Escala de Afrontamiento Ante la Ansiedad e Incertidumbre Pre-Examen (20 items)

\begin{tabular}{llrrrrrr}
\hline & \multicolumn{1}{c}{ Sexo } & $\mathrm{N}$ & Media & $\begin{array}{c}\text { Desviación } \\
\text { estándar }\end{array}$ & $\mathrm{t}$ & $\mathrm{gl}$ & $\mathrm{p}$ \\
\hline Orientación a la & Femenino & 155 & 30,8323 & 5,92466 & 1,617 & 228 &, 107 \\
Tarea & Masculino & 75 & 29,5067 & 5,61940 & & & \\
Evitación & Femenino & 155 & 18,4387 & 4,15618 &,- 394 & 228 &, 694 \\
& Masculino & 75 & 18,6800 & 4,75099 & & & \\
Búsqueda de apoyo & Femenino & 155 & 10,3613 & 3,10191 & 1,168 & 228 &, 244 \\
Instrumental & Masculino & 75 & 9,8533 & 3,07428 & & &, 004 \\
& & & & & & & \\
Búsqueda de apoyo & Femenino & 155 & 12,9355 & 4,66220 & 2,948 & 228 & \\
Emocional & Masculino & 75 & 11,0533 & 4,27087 & & & \\
\hline
\end{tabular}

En la Tabla 7 se muestran los puntajes brutos diferenciados por sexo de cada uno de los factores asociados a sus respectivos percentiles.

Tabla 7.

Baremos de la Escala de Afrontamiento Ante la Ansiedad e Incertidumbre Pre-Examen (20 items)

\begin{tabular}{ccccccccc}
\hline & \multicolumn{2}{c}{$\begin{array}{c}\text { Orientación a la } \\
\text { Tarea }\end{array}$} & \multicolumn{2}{c}{ Evitación } & \multicolumn{2}{c}{$\begin{array}{c}\text { Búsqueda de Apoyo } \\
\text { Instrumental }\end{array}$} & \multicolumn{2}{c}{$\begin{array}{c}\text { Búsqueda de Apoyo } \\
\text { Emocional }\end{array}$} \\
\cline { 2 - 9 } Percentiles & Mujer & Hombre & Mujer & Hombre & Mujer & Hombre & Mujer & Hombre \\
\hline 10 & 22 & 22 & 14 & 13 & 6 & 6 & 8 & 6 \\
20 & 24 & 24 & 15 & 14 & 8 & 7 & 9 & 8 \\
30 & 28 & 26 & 16 & 17 & 8 & 8 & 10 & 9 \\
40 & 30 & 28 & 17 & 18 & 9 & 9 & 11 & 10 \\
50 & 31 & 29 & 18 & 19 & 10 & 10 & 12 & 11 \\
60 & 33 & 31 & 20 & 20 & 11 & 11 & 14 & 11 \\
70 & 35 & 33 & 21 & 21 & 12 & 11 & 16 & 13 \\
80 & 37 & 35 & 22 & 22 & 13 & 12 & 18 & 15 \\
90 & 38 & 37 & 24 & 25 & 14 & 14 & 20 & 17 \\
\hline
\end{tabular}

\section{Discusión}

La presente investigación tenía como objetivo indagar las propiedades psicométricas de la adaptación argentina de la escala de afrontamiento ante la ansiedad e incertidumbre COPEAU-A 
(Stöber, 2004; Heredia, Piemontesi, Furlan \& Pérez, 2008) en estudiantes chilenos de nivel universitario. Los resultados obtenidos avalan las propiedades psicométricas del instrumento.

En cuanto a la estructura factorial del instrumento, se verificó el ajuste de cinco modelos. En primer lugar, se constata los mejores indicadores de ajuste en el modelo de cuatro factores oblicuos y 20 ítems (orientación a la tarea, búsqueda de apoyo por razones instrumentales, búsqueda de apoyo por razones emocionales y evitación), propuesto por Domínguez-Lara y Merino-Soto (2016) en Perú. Respecto a esta versión del instrumento se verificó la invarianza estructural para la muestra de hombres y mujeres, lo cual permite constatar su adecuación teórica y empírica para evaluar a ambos grupos. A su vez, presenta en las 4 subescalas que lo componen una apropiada confiabilidad, evidenciando valores omegas de McDonald superiores a ,70 en todas ellas.

El afrontamiento ante la ansiedad en las evaluaciones académicas se hace fundamental, ya que marca la diferencia en la capacidad de adaptarse a las diversas exigencias académicas y sociales que viven los estudiantes universitarios en su día a día (Castillo, 2016). La carga académica, las evaluaciones, presentaciones, la relación con docentes, la adaptación al medio universitario, la planificación del tiempo, gastos económicos y las expectativas personales aparecen como los principales estresores de la realidad universitaria (Cabanach, Souto-Gestal, \& Franco, 2016).

Las posibles dificultades que atraviesan los estudiantes al momento de preparar y rendir evaluaciones académicas se deben identificar a tiempo, para que así se tomen las remediales de manera pronta y oportuna, ya sea con intervenciones individuales y grupales, que consideren las estrategias de afrontamiento ante las evaluaciones, $y$, así, ser una ayuda en pro de mejorar la preparación y rendiciones de las mismas, ya no solo pensando en el rendimiento académico en sí, sino, que también considerando aspectos emocionales. Se hace importante entregar estrategias que lleven al afrontamiento y regulación de situaciones complejas que los estudiantes pudieran encontrar en cursos superiores, prácticas y en la vida laboral a través de intervenciones psicoeducativas realizadas dentro del programa de estudios de cada carrera (Santos-Ruiz, Fernández-Pascual, Reig-Ferrer, Riquelme-Ros, Montero-López, Peralta-Ramírez, 2019).

Estas intervenciones de fortalecimiento de las estrategias de afrontamiento para situaciones de estrés pueden ser, ya sea, centradas en la orientación a la tarea, en búsqueda de apoyo por razones instrumentales y en búsqueda de apoyo por razones emocionales, desarrolladas desde un enfoque validado y eficaz como lo es el cognitivo-conductual, así como considerar incluir técnicas de atención de plena para aliviar el malestar psicológico (Roulston, Montgomery, Campbell, \& Davidson, 2018).

Por estas razones, es que se hace perentorio que en las diferentes instituciones educativas se pueda contar con instrumentos validados y el uso de baremos específicos que permitan identificar de manera confiable estos constructos, para así diseñar programas e intervenciones psicoeducativas que favorezcan el fortalecimiento de recursos personales, que lleven a la mejora en la calidad de vida, bienestar dentro de la institución y al rendimiento académico de los estudiantes universitarios (Dominguez-Lara, Fernández-Arata, Manrique-Millones, AlarcónParco, Díaz-Peñaloza, 2017).

Dentro de las limitaciones de la investigación se pueden identificar las características sociodemográficas y el tamaño de la muestra, ya que solamente se consideraron estudiantes del norte de Chile que inician sus estudios universitarios. Para próximos estudios sería importante aumentar la muestra e incluir estudiantes de diferentes niveles académicos, socioeconómicos y de comunas del país, para así evaluar diferencias entre las diversas poblaciones universitarias. Otro aspecto a considerar sería determinar la invarianza factorial del instrumento en diferentes países de la región en donde ya se han realizado estudios similares. 


\section{Referencias}

Angelucci, LT., Cañoto, Y., Hernández, MJ. (2017). Influencia del estilo de vida, el sexo, la edad y el imc sobre la salud física y psicológica en jóvenes universitarios. Avances en Psicología Latinoamericana, 35(3), 531-546. http://doi.org/10.12804/revistas.urosario.edu.co/apl/a.4454

Arana, F. \& Furlan, L. (2015). Groups of perfectionists, test anxiety, and pre-exam coping in Argentine students. Personality and Individual Differences, 90, 169-173. https://doi.org/10.1016/j.paid.2015.11.001

Ato, M., López, JJ., \& Benavente, A. (2013). Un sistema de clasificación de los diseños de investigación en psicología. Anales de Psicología, 29(3),1038-1059.

Byrne, B.M. (2008). Testing for multigroup equivalence of a measuring instrument: A walk through the process. Psicothema, 20(4), 872-882.

Brown, T. (2015). Confirmatory Factor Analysis for Applied Research. The Guilford Press

Cabanach, RG., \& Souto-Gestal, A., \& Franco, V. (2016). Escala de Estresores Académicos para la evaluación de los estresores académicos en estudiantes universitarios. Revista Iberoamericana de Psicología y Salud, 7(2),41-50.

Carver, CS., \& Scheier, MF. (1995). Situational coping and coping dispositions in a stressful encounter. Journal of Personality and Social Psychology.

Carver, C.S., Scheier, MF. \& Weintraub, J.K. (1989) Assesing Coping Strategies: A Theoretically Based Approach. Journal of Personality and Social Psychology, 56, 267- 283.

Castillo, C., Chacón, T., \& Díaz-Véliz, G. (2016). Ansiedad y fuentes de estrés académico en estudiantes de carreras de la salud. Investigación en educación médica, 5(20), 230237. https://doi.org/10.1016/j.riem.2016.03.001

Cheung, G.W., \& Rensvold, RB. (2002). Evaluating goodness-of-fit indexes for testing measurement invariance. Structural Equation Modeling: A Multidisciplinary Journal, 9(2), 233-255. https://doi.org/10.1 207/S15328007SEM0902_5

Domínguez-Lara, S. (2018a). Afrontamiento ante la ansiedad pre-examen y autoeficacia académica en estudiantes de ciencias de la salud. Educación Médica, 19(1), 39-42. https://doi.org/10.1016/j.edumed.2016.07.007

Domínguez-Lara, S. (2018b). Evidencias convergente y discriminante del TAI-Estado: Influencia de las estrategias de afrontamiento pre-examen. Revista Digital de Investigación en Docencia Universitaria, 12(1), 237-255. http://doi.org/10.19083/ridu.2018.547

Domínguez-Lara, S. (2018c). Procrastinación académica, afrontamiento de la ansiedad preexamen y rendimiento académico en estudiantes de psicología: análisis preliminar. Cultura: Revista de La Asociación de Docentes de La USMP, 32, 181-193. http://doi.org/10.24265/cultura.2017.v31.10

Domínguez-Lara, S., Fernández-Arata, M., Manrique-Millones, D., Alarcón-Parco, D., \& DíazPeñaloza, M. (2017). Datos normativos de una escala de agotamiento emocional académico en estudiantes universitarios de psicología de Lima (Perú). Educación Médica, 19(3), 246 - 255. http://doi.org/10.1016/j.edumed.2017.09.002

Domínguez-Lara, S. \& Merino-Soto, C. (2016). Análisis Estructural de la Escala de Afrontamiento ante la Ansiedad e Incertidumbre Pre-examen (COPEAU) en universitarios peruanos. Revista Digital de Investigación en Docencia Universitaria, 10(2), 32-47. doi: http://doi.org/10.19083/ridu.10.474

Domínguez-Lara, S. \& Merino-Soto, C. (2018). Versión breve de la Escala de afrontamiento ante la ansiedad e incertidumbre preexamen (COPEAU) en universitarios peruanos. Educación Médica, 19 (2), 67-72. https://doi.org/10.1016/j.edumed.2017.04.011

Fernández-Castillo, A., \& Caurcel, M. J. (2015). State test-anxiety, selective attention and concentration in university students. International Journal of Psychology, 50(4). https://doi.org/10.1002 / ijop.12092.

Heredia, D., Piemontesi, S., Furlan, L. \& Perez, E. (2008). Adaptacion de la escala de afrontamiento ante la ansiedad e incertidumbre pre-examen (COPEAU). Avaliacao Psicologica, 7(1), 1-9. 
Hu, L. \& Bentler, P. (1999). Cutoff criteria for fit indexes in covariance structure analysis: Conventional criteria versus new alternatives. Structural Equation Modeling: A Multidisciplinary Journal, 6(1), 1-55. https://doi.org/10.1080/10705519909540118

Iacobucci, D. (2010). Structural equations modeling: Fit indices, sample size, and advanced topics. Journal of Consumer Psychology, 20(1), 90-98. doi: http://doi.org/10.1016/j.jcps.2009.09.003

JASP Team (2020). JASP (Version 0.14) [Computer software].

Kline, R. (2005). Principles and practice of structural equation modeling. Guilford

Lazarus, R. S. \& Folkman, S. (1984) Stress, Appraisal and Coping. New York: Springer Publishing Company.

Oermann, M. H., \& Gaberson, K. B. (2009). Evaluation and testing in nursing education (3rd ed.). New York: Springer Publishing Company.

R Core Team (2020). $R$ : A language and environment for statistical computing. R Foundation for Statistical Computing, Vienna, Austria. URL https://www.R-project.org/.

Rost, D. H., \& Schermer, F. J. (1997). Differentielles Leistungsangst Inventar (DAI): Handbuch [Differential Performance Anxiety Inventory: Manual]. Frankfurt/Main, Swets Test Services, Germany.

Roulston, A., Montgomery, L., Campbell, A., \& Davidson, G. (2018). Exploring the impact of mindfulnesss on mental wellbeing, stress and resilience of undergraduate social work students. Social Work Education, 37(2), 157-172. https://doi.org/10.1080/02615479.2017.1388776

Santabárbara, J. (2019). Afrontamiento ante la ansiedad e incertidumbre preexamen de bioestadística en estudiantes de Grado en Medicina. FEM: Revista de la Fundación Educación Médica, 22(5), 207-210

Santos-Ruiz, A., Fernández-Pascual, M. D., Reig-Ferrer, A., Riquelme-Ros, L., Montero-López, E., \& Peralta-Ramírez, M. I. (2019). Valoración del estrés percibido y de las necesidades y demandas de intervención psicoeducativa para su manejo eficaz en estudiantes universitarios. En R. Roig-Vila (Ed.), Investigación e innovación en la Enseñanza Superior. Nuevos contextos, nuevas ideas (pp. 409-416). Barcelona: Octaedro.

Stöber, J. (2004). Dimensions of test anxiety: relations to ways of coping with pre-exam anxiety and uncertainty. Anxiety, Stress, and Coping, 17 (3), 213-226.

Zeidner, M. (1995). Adaptive coping with test situations: A review of the literature. Educational Psychologist, 30, 123-133. 